\title{
Into the depths of the heart: The case for transthoracic intracardiac lines placed during cardiac surgery
}

\author{
Ibrahim Abdullah, MD
}

See related article on pages 1212-3.

In the current issue of The Journal of Thoracic and Cardiovascular Surgery, Pratap and colleagues ${ }^{1}$ from the Royal Children's Hospital in Melbourne present a retrospective review of complications of intrathoracic lines placed during cardiac surgery at their institution through an impressive span from 2005 to 2013. Quite aptly, they note the paucity of data regarding complications of transthoracic intracardiac lines. In fact, despite the limited number of series that have been published focusing on transthoracic intracardiac line complications, there appears to be an insinuation of their frequent use at the respective institutions. For example, Gold and colleagues ${ }^{2}$ report the use of 6690 transthoracic intracardiac monitoring catheters in 5666 patients during a 10 -year period. The other series, by Flori and colleagues, ${ }^{3}$ reports the use of 523 catheters in 351 patients during a 1-year period. Does this signify similar frequency of use of such lines across institutions?

There is no doubt that transthoracic intracardiac lines have an important role in the postoperative management of critically ill pediatric cardiac patients. For example, as the authors note, a right atrial (RA) line may be preferred over a neck central line in a patient with a single-ventricle heart to avoid risk to a future Glenn pathway. A left atrial line may be helpful in a patient who has undergone a complex arterial switch operation. A rise in the left atrial pressure may portend left ventricular dysfunction due to coronary malperfusion. A pulmonary arterial line may help guide therapy for a neonate with significant pulmonary reactivity after a total anomalous pulmonary venous return repair. Despite their great utility, however, transthoracic intracardiac lines do not come without risk.

\footnotetext{
From the Division of Pediatric Cardiothoracic Surgery, Children's Hospital and Medical Center, Omaha, Neb; and the Department of Surgery, University of Nebraska Medical Center, Omaha, Neb.

Disclosures: Author has nothing to disclose with regard to commercial support.

Received for publication Dec 20, 2014; accepted for publication Dec 20, 2014; available ahead of print Jan 29, 2015.

Address for reprints: Ibrahim Abdullah, MD, Division of Pediatric Cardiothoracic Surgery, Children's Hospital and Medical Center, Omaha, Nebraska, Department of Surgery, University of Nebraska Medical Center, 8200 Dodge St, Omaha, NE 68114 (E-mail: iabdullah@childrensomaha.org).

J Thorac Cardiovasc Surg 2015;149:1214

$0022-5223 / \$ 36.00$

Copyright (C) 2015 by The American Association for Thoracic Surgery

http://dx.doi.org/10.1016/j.jtcvs.2014.12.052
}

Pratap and colleagues ${ }^{1}$ mention that of 1404 transthoracic lines, complications of migration (or premature removal), bleeding, thrombosis, occlusion, and failed removal were seen in $30(2.1 \%)$. Of these complications, the overwhelming majority $(\mathrm{n}=17)$ occurred in the category of migration or premature removal. Within the migration or premature removal category, the majority of complications (12 of 17) were associated with RA lines, although the number of RA lines placed was less than the numbers of left atrial and pulmonary arterial lines placed in their series. Furthermore, 5 of these 12 RA line migrations necessitated a return to the operating room, with 2 of the 5 patients requiring ECMO support. Pratap and colleagues ${ }^{1}$ also describe 2 different fixation techniques for the RA lines. In one technique, there is additional extracardiac reinforcement (as shown in their Figure 1). It is humbling to note that 5 of the 12 RA migrations occurred in the "reinforced" lines. One is left to wonder whether there is a benefit to the described reinforced technique. Overall, Pratap and colleagues ${ }^{1}$ report that 9 cases of line-related complications required surgical intervention, and 8 of these $(89 \%)$ were for life-threatening bleeding. Although the overall complication rate of transthoracic intracardiac lines appears to be low, the chance that any complication that occurs will be devastating remains high.

Given the possibility of a devastating complication, perhaps a deeper question to ask as a community of pediatric cardiac surgeons is whether the routine placement of transthoracic intracardiac lines is warranted? Perhaps a more selective approach ought to be used. If we decide to use these lines, do we need to come up with novel reinforcement techniques? Do we need to enact more sophisticated protocols for their use and removal? Given the scarcity of published reports on the incidence of complications of such lines, Pratap and colleagues ${ }^{1}$ have to be commended for sharing their experience and providing a stimulus for the pediatric cardiac surgical community to reflect on and share their own experiences.

\footnotetext{
References

1. Pratap H, Millar J, Butt W, d'Udekem Y. Complications of intrathoracic lines placed during cardiac surgery. J Thorac Cardiovasc Surg. 2015;149: 1212-3.

2. Gold JP, Jonas RA, Lang P, Elixson EM, Mayer JE, Castaneda AR. Transthoracic intracardiac monitoring lines in pediatric surgical patients: a ten-year experience. Ann Thorac Surg. 1986;42:185-91.

3. Flori HR, Johnson LD, Hanley FL, Fineman JR. Transthoracic intracardiac catheters in pediatric patients recovering from congenital heart defect surgery: associated complications and outcomes. Crit Care Med. 2000;28:2997-3001.
} 